\title{
Influence of INM on Nutrient Uptake of Cotton in Dry Land Condition
}

\author{
Megha S. Khambalkar, V.V. Gabhane and Shilpa V. Khambalkar* \\ Dr. Panjabrao Deshmukh Krishi Vidyapeeth, Akola, India \\ *Corresponding author
}

\begin{tabular}{|c|c|}
\hline & A B S T R A C T \\
\hline $\begin{array}{l}\text { K e y w o r d s } \\
\text { Cotton, Integrated } \\
\text { nutrient } \\
\text { management, } \\
\text { Biofertilizers. }\end{array}$ & \multirow{3}{*}{$\begin{array}{l}\text { An experiment "Nutrient dynamics and productivity of Cotton in Vertisols under } \\
\text { integrated nutrient management" was conducted during kharif } 2014 \text { at Research field of } \\
\text { AICRP for Dryland Agriculture, Dr. Panjabrao Deshmukh Krishi Vidyapeeth, Akola to } \\
\text { study the effect of integrated nutrient management on Yield of cotton and Nutrient uptake } \\
\text { by cotton. The experiment was conducted with ten treatments and three replications laid } \\
\text { out in a randomized block design. The result of present experiment revealed that the } \\
\text { integrated application of } 50 \% \mathrm{~N} \text { through gliricidia }+50 \% \mathrm{~N} \text { through inorganics }+ \\
\text { biofertilizers+ } 100 \% \mathrm{P}+25 \mathrm{~kg} \mathrm{~K} \mathrm{~K}^{-1} \text { resulted in improvement in soil fertility, nutrient } \\
\text { uptake and yield of cotton grown in Vertisols under rainfed conditions. }\end{array}$} \\
\hline Article Info & \\
\hline $\begin{array}{l}\text { Accepted: } \\
\text { 27 June } 2017 \\
\text { Available Online: } \\
\text { 10 August } 2017\end{array}$ & \\
\hline
\end{tabular}

\section{Introduction}

Cotton is one of the most important cash crops which play a key role in economy and social affairs. The nutrients removal by crops is generally more than that supplied through chemical fertilizer and this negative balance over the years led to low fertility status of soil, which resulted in decline in the crop yields and also the heavy use of chemical fertilizers which are increasing day by day. The indiscriminate use of these chemical fertilizers is prone to several environmental problems like deterioration of soil heath and contamination of natural resources.

Adequate and timely application of organic and also inorganic fertilizer is most essential for proper growth of the crop. Nutritional stresses and imbalance affect vegetative as well as reproductive growth that ultimately lower down the average seed cotton yields as well as fibre and seed quality.

\section{Materials and Methods}

With a view to study the "Nutrient Dynamics and Productivity of Cotton in Vertisols under Integrated Nutrient Management", a field experiment was initiated on the research field of AICRP for Dryland Agriculture, Dr. PDKV, Akola. The present study was undertaken during 2014-15 with the cotton crop.

This experiment was conducted with ten treatments and three replications laid out in a randomized block design, treatment details are as follows $\mathrm{T}_{1}$ - Control, $\mathrm{T}_{2}-100 \% \mathrm{NP}$ (50:25:00 NPK kg ha $\left.{ }^{-1}\right), \mathrm{T}_{3}-100 \% \mathrm{NP}+$ biofertilizers, $\mathrm{T}_{4}-100 \% \mathrm{~N}$ through $\mathrm{FYM}+$ biofertilizers, $\mathrm{T}_{5}-100 \% \mathrm{~N}$ through gliricidia + biofertilizers, $\mathrm{T}_{6}-100 \% \mathrm{NP}+25 \mathrm{~kg} \mathrm{~K} \mathrm{ha}^{-1}, \mathrm{~T}_{7^{-}}$ $100 \% \mathrm{NP}+25 \mathrm{~kg} \mathrm{~K} \mathrm{ha}^{-1}+$ biofertilizers, $\mathrm{T}_{8^{-}}$ $50 \% \mathrm{~N}$ through gliricidia $+50 \% \mathrm{~N}$ through inorganics $+100 \% \mathrm{P}, \mathrm{T}_{9}-50 \% \mathrm{~N}$ through 
gliricidia $+50 \% \mathrm{~N}$ through inorganics + biofertilizer $+100 \%$ P. The seed was sown@ $10 \mathrm{~kg} / \mathrm{ha}$ with the recommended dose of fertilizer i.e. 50:25:0 NPK kg/ha.

Full quantity of the recommended dose of phosphorus was applied as a basal dose through single super phosphate. Nitrogen through urea in two-spilt application, half at the sowing and half at 35 DAS to cotton. The spacing was $60 \mathrm{~cm}$ for row to row and $30 \mathrm{~cm}$ for plant to plant. The seed treatment given was Azotobacter and PSB @ $25 \mathrm{~g} \mathrm{~kg}^{-1}$ seed. The soil samples were collected plot wise after harvest of cotton crop and analyzed for various physical and chemical properties of the soil. Similarly plant samples were also collected at harvest stage, the seed cotton and cotton stalk yield was also recorded.

\section{Results and Discussion}

The data indicated that the significantly higher $\mathrm{N}$ uptake $\left(28.05 \mathrm{~kg} \mathrm{ha}^{-1}\right.$ and $10.31 \mathrm{~kg}$ $\mathrm{ha}^{-1}$ ) by cotton seed and stalk was observed with the application of $50 \% \mathrm{~N}$ through gliricidia $+50 \% \mathrm{~N}$ through inorganics + biofertilizers $+100 \% \mathrm{P}+25 \mathrm{~kg} \mathrm{~K} \mathrm{ha}^{-1}\left(\mathrm{~T}_{10}\right)$ and also significantly highest total $\mathrm{N}$ uptake (38.36 $\mathrm{kg} \mathrm{ha}^{-1}$ ) by cotton was observed in treatment $\mathrm{T}_{10}$. The lowest $\mathrm{N}$ and $\mathrm{P}$ uptake by cotton seed and stalk $\left(6.35 \mathrm{~kg} \mathrm{ha}^{-1}\right.$ and $2.58 \mathrm{~kg}$ $\mathrm{ha}^{-1}$ ) was observed in treatment $\mathrm{T}_{1}$ i.e. control.

Table.1 Effect of INM on nitrogen uptake by cotton

\begin{tabular}{|c|c|c|c|c|}
\hline \multicolumn{2}{|r|}{ Treatments } & \multicolumn{3}{|c|}{$\begin{array}{l}\text { Nitrogen uptake } \\
\quad\left(\mathrm{kg} \mathrm{ha}^{-1}\right)\end{array}$} \\
\hline & & \multirow{2}{*}{$\begin{array}{l}\text { Seed } \\
6.35\end{array}$} & \multirow{2}{*}{$\begin{array}{l}\text { Stalk } \\
2.58\end{array}$} & \multirow{2}{*}{$\begin{array}{l}\text { Total } \\
8.93\end{array}$} \\
\hline $\mathrm{T}_{1}$ & Control & & & \\
\hline $\mathrm{T}_{2}$ & $100 \%$ NP (50:25:00 NPK kg ha $\left.{ }^{-1}\right)$ & 10.73 & 4.16 & 14.88 \\
\hline $\mathrm{T}_{3}$ & $100 \%$ NP + biofertilizers & 11.58 & 4.87 & 16.45 \\
\hline $\mathrm{T}_{4}$ & $100 \% \mathrm{~N}$ through FYM + biofertilizers & 8.58 & 2.81 & 11.39 \\
\hline $\mathrm{T}_{5}$ & $100 \% \mathrm{~N}$ through gliricidia+ biofertilizers & 8.67 & 3.18 & 11.85 \\
\hline $\mathrm{T}_{6}$ & $100 \% \mathrm{NP}+25 \mathrm{~kg} \mathrm{~K} \mathrm{ha}{ }^{-1}$ & 15.57 & 5.63 & 21.21 \\
\hline $\mathrm{T}_{7}$ & $100 \% \mathrm{NP}+25 \mathrm{~kg} \mathrm{~K} \mathrm{ha}^{-1}+$ biofertilizers & 21.23 & 8.29 & 29.52 \\
\hline $\mathrm{T}_{8}$ & $\begin{array}{l}50 \% \mathrm{~N} \text { through gliricidia }+50 \% \mathrm{~N} \text { through inorganics+ } \\
100 \% \mathrm{P}\end{array}$ & 13.76 & 4.95 & 18.71 \\
\hline $\mathrm{T}_{9}$ & $\begin{array}{l}50 \% \mathrm{~N} \text { through gliricidia }+50 \% \mathrm{~N} \text { through inorganics+ } \\
100 \% \mathrm{P}+\text { biofertilizers }\end{array}$ & 15.10 & 5.96 & 21.06 \\
\hline $\mathrm{T}_{10}$ & $\begin{array}{l}50 \% \mathrm{~N} \text { through gliricidia }+50 \% \mathrm{~N} \text { through inorganics+ } \\
100 \% \mathrm{P}+\text { biofertilizers }+25 \mathrm{~kg} \mathrm{Kg} \mathrm{ha}^{-1}\end{array}$ & 28.05 & 10.31 & 38.36 \\
\hline & $\mathrm{SE}(\mathrm{m})+$ & 2.25 & 0.81 & 3.03 \\
\hline & $\mathrm{CD}$ at $5 \%$ & 6.70 & 2.41 & 8.99 \\
\hline
\end{tabular}


Table.2 Effect of INM on phosphorus uptake by cotton

\begin{tabular}{|c|c|c|c|c|}
\hline \multirow{2}{*}{\multicolumn{2}{|c|}{ Treatments }} & \multicolumn{3}{|c|}{$\begin{array}{l}\text { Phosphorus uptake } \\
\qquad\left(\mathrm{kg} \mathrm{ha}^{-1}\right)\end{array}$} \\
\hline & & Seed & Stalk & Total \\
\hline $\mathrm{T}_{1}$ & Control & 0.95 & 1.31 & 2.26 \\
\hline $\mathrm{T}_{2}$ & $100 \%$ NP $\left(50: 25: 00\right.$ NPK kg ha $\left.{ }^{-1}\right)$ & 1.66 & 2.51 & 4.17 \\
\hline $\mathrm{T}_{3}$ & $100 \% \mathrm{NP}+$ biofertilizers & 1.79 & 2.81 & 4.59 \\
\hline $\mathrm{T}_{4}$ & $100 \% \mathrm{~N}$ through FYM + biofertilizers & 1.14 & 1.65 & 2.80 \\
\hline $\mathrm{T}_{5}$ & $100 \% \mathrm{~N}$ through gliricidia+ biofertilizers & 1.23 & 1.80 & 3.03 \\
\hline $\mathrm{T}_{6}$ & $100 \% \mathrm{NP}+25 \mathrm{~kg} \mathrm{~K} \mathrm{ha}^{-1}$ & 2.30 & 3.65 & 5.95 \\
\hline $\mathrm{T}_{7}$ & $100 \% \mathrm{NP}+25 \mathrm{~kg} \mathrm{~K} \mathrm{ha}^{-1}+$ biofertilizers & 3.17 & 5.27 & 8.44 \\
\hline $\mathrm{T}_{8}$ & $\begin{array}{l}50 \% \mathrm{~N} \text { through gliricidia }+50 \% \mathrm{~N} \text { through } \\
\text { inorganics }+100 \% \mathrm{P}\end{array}$ & 2.06 & 3.25 & 5.30 \\
\hline $\mathrm{T}_{9}$ & $\begin{array}{l}50 \% \mathrm{~N} \text { through gliricidia }+50 \% \mathrm{~N} \text { through inorganics+ } \\
100 \% \mathrm{P}+\text { biofertilizers }\end{array}$ & 2.31 & 3.73 & 6.05 \\
\hline $\mathrm{T}_{10}$ & $\begin{array}{l}50 \% \mathrm{~N} \text { through gliricidia }+50 \% \mathrm{~N} \text { through inorganics } \\
+ \text { biofertilizers }+100 \% \mathrm{P}+25 \mathrm{~kg} \mathrm{~K}^{-1}\end{array}$ & 4.02 & 6.70 & 10.72 \\
\hline & $\mathrm{SE}(\mathrm{m})+$ & 0.31 & 0.51 & 0.82 \\
\hline & CD at $5 \%$ & 0.92 & 1.52 & 2.43 \\
\hline
\end{tabular}

Table.3 Effect of INM on potassium uptake by cotton

\begin{tabular}{|c|c|c|c|c|}
\hline \multirow{2}{*}{\multicolumn{2}{|c|}{ Treatments }} & \multicolumn{3}{|c|}{$\begin{array}{c}\text { Potassium uptake } \\
\left(\mathrm{kg} \mathrm{ha}^{-1}\right)\end{array}$} \\
\hline & & \multirow{2}{*}{$\begin{array}{c}\text { Seed } \\
1.23\end{array}$} & \multirow{2}{*}{$\begin{array}{c}\text { Stalk } \\
5.04 \\
\end{array}$} & \multirow{2}{*}{$\begin{array}{c}\text { Total } \\
6.27 \\
\end{array}$} \\
\hline $\mathrm{T}_{1}$ & Control & & & \\
\hline $\mathrm{T}_{2}$ & $100 \%$ NP (50:25:00 NPK kg ha $\left.{ }^{-1}\right)$ & 1.80 & 7.65 & 9.44 \\
\hline $\mathrm{T}_{3}$ & $100 \% \mathrm{NP}+$ biofertilizers & 2.14 & 8.52 & 10.66 \\
\hline $\mathrm{T}_{4}$ & $100 \% \mathrm{~N}$ through FYM + biofertilizers & 1.48 & 6.31 & 7.79 \\
\hline $\mathrm{T}_{5}$ & $100 \% \mathrm{~N}$ through gliricidia + biofertilizers & 1.57 & 6.72 & 8.29 \\
\hline $\mathrm{T}_{6}$ & $100 \% \mathrm{NP}+25 \mathrm{~kg} \mathrm{~K} \mathrm{ha}^{-1}$ & 2.46 & 10.02 & 12.48 \\
\hline $\mathrm{T}_{7}$ & $100 \% \mathrm{NP}+25 \mathrm{~kg} \mathrm{~K} \mathrm{ha}^{-1}+$ biofertilizers & 3.35 & 13.94 & 17.29 \\
\hline $\mathrm{T}_{8}$ & $\begin{array}{l}50 \% \mathrm{~N} \text { through gliricidia }+50 \% \mathrm{~N} \text { through } \\
\text { inorganics }+100 \% \mathrm{P}\end{array}$ & 2.18 & 9.03 & 11.21 \\
\hline $\mathrm{T}_{9}$ & $\begin{array}{l}50 \% \mathrm{~N} \text { through gliricidia }+50 \% \mathrm{~N} \text { through } \\
\text { inorganics }+100 \% \mathrm{P}+\text { biofertilizers }\end{array}$ & 2.41 & 10.09 & 12.50 \\
\hline $\mathrm{T}_{10}$ & $\begin{array}{l}50 \% \mathrm{~N} \text { through gliricidia }+50 \% \mathrm{~N} \text { through } \\
\text { inorganics + biofertilizers }+100 \% \mathrm{P}+25 \mathrm{~kg} \mathrm{~K}^{-1}\end{array}$ & 4.27 & 17.79 & 22.06 \\
\hline & $\mathrm{SE}(\mathrm{m}) \pm$ & 0.34 & 1.41 & 1.74 \\
\hline & $\mathrm{CD}$ at $5 \%$ & 1.00 & 4.18 & 5.18 \\
\hline
\end{tabular}




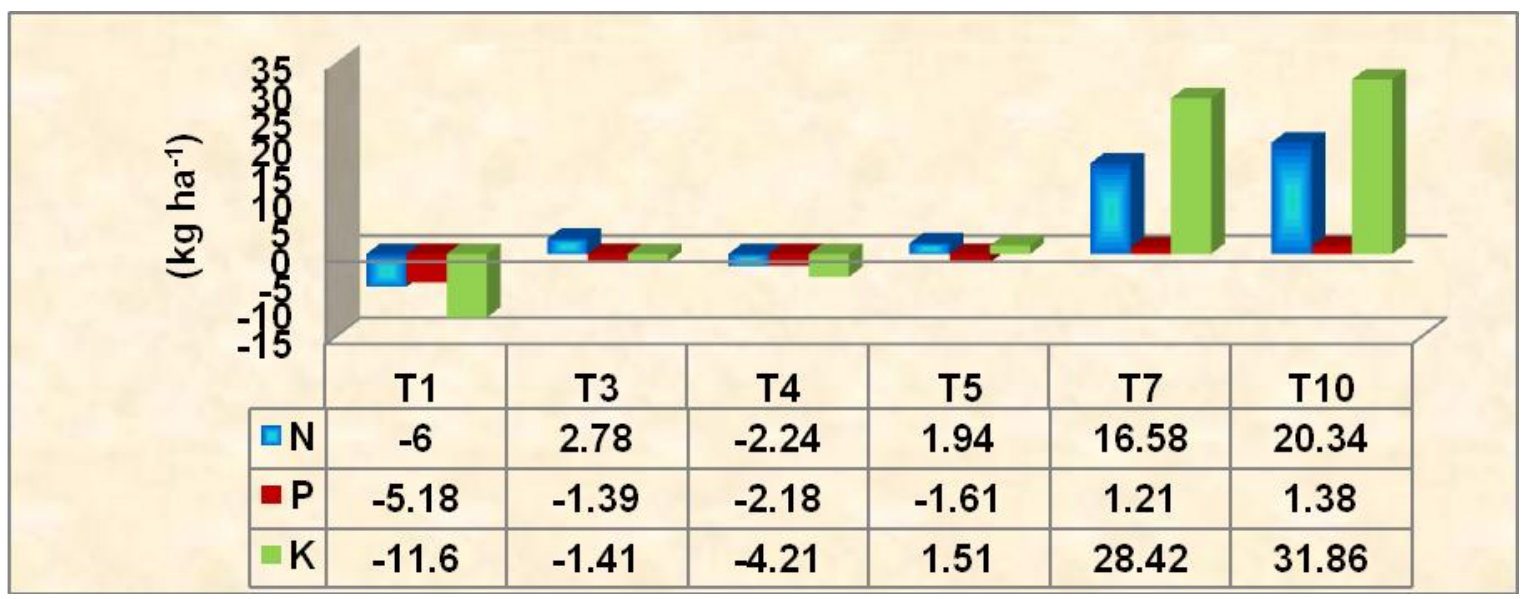

The uptake of $\mathrm{N}$ increased due to the combined application of $50 \% \mathrm{~N}$ through gliricidia $+50 \% \mathrm{~N}$ through inorganics + $100 \% \mathrm{P}+25 \mathrm{~kg} \mathrm{~K}+$ biofertilizers which increase the concentration of $\mathrm{N}$ in seed and stalk.

Similarly higher $\mathrm{P}$ uptake $\left(4.02 \mathrm{~kg} \mathrm{ha}^{-1}\right.$ and $6.70 \mathrm{~kg} \mathrm{ha}^{-1}$ ) by cotton seed and stalk was observed with the application of $50 \% \mathrm{~N}$ through gliricidia $+50 \% \mathrm{~N}$ through inorganics + biofertilizers $+100 \% \mathrm{P}+25 \mathrm{~kg} \mathrm{~K}$ $\mathrm{ha}^{-1}\left(\mathrm{~T}_{10}\right)$ and it was on par with the application of $100 \% \mathrm{NP}+25 \mathrm{~kg} \mathrm{~K} \mathrm{ha}^{-1}+$ biofertilizers $\left(\mathrm{T}_{7}\right)$. The lowest $\mathrm{P}$ uptake by cotton seed and stalk $\left(0.95 \mathrm{~kg} \mathrm{ha}^{-1}\right.$ and $1.31 \mathrm{~kg}$ $\mathrm{ha}^{-1}$ was observed in treatment $\mathrm{T}_{1}$ i.e. control. The significantly higher total $\mathrm{P}$ uptake $(10.72$ $\mathrm{kg} \mathrm{ha}^{-1}$ ) by cotton was observed in treatment $\mathrm{T}_{10}$ and it was found to be on par with the treatment $\mathrm{T}_{7}$ i.e. $100 \% \mathrm{NP}+25 \mathrm{~kg} \mathrm{~K} \mathrm{ha}^{-1}+$ biofertilizers. The lowest $\mathrm{P}$ uptake by cotton was observed in treatment $\mathrm{T}_{1}$ i.e. control $(2.26$ $\mathrm{kg} \mathrm{ha}^{-1}$ ).

Significantly higher K uptake $\left(4.27 \mathrm{~kg} \mathrm{ha}^{-1}\right.$, $17.79 \mathrm{kgha}^{-1}$ and $22.06 \mathrm{~kg} \mathrm{ha}^{-1}$ ) by cotton seed, stalk and cotton was observed with the application of $50 \% \mathrm{~N}$ through gliricidia + $50 \% \mathrm{~N}$ through inorganics + biofertilizers+ $100 \% \mathrm{P}+25 \mathrm{~kg} \mathrm{~K} \mathrm{ha}^{-1}\left(\mathrm{~T}_{10}\right)$ and it was found to be on par with application of $100 \% \mathrm{NP}+$
$25 \mathrm{~kg} \mathrm{~K} \mathrm{ha}{ }^{-1}+$ biofertilizers $\left(\mathrm{T}_{7}\right)$. The lowest $\mathrm{K}$ uptake $\left(1.23 \mathrm{~kg} \mathrm{ha}^{-1}, 5.04 \mathrm{~kg} \mathrm{ha}^{-1}\right.$ and 6.27 $\mathrm{kg} \mathrm{ha}^{-1}$ ) by cotton seed, stalk and cotton was observed in treatment $\mathrm{T}_{1}$ i.e. control.

In conclusion the significantly higher $\mathrm{N}$ uptake $\left(28.05 \mathrm{~kg} \mathrm{ha}^{-1}\right)$ by cotton seed was observed with the application of $50 \% \mathrm{~N}$ through gliricidia $+50 \% \mathrm{~N}$ through inorganics + biofertilizers $+100 \% \mathrm{P}+25 \mathrm{~kg} \mathrm{~K}$ $\mathrm{ha}^{-1}\left(\mathrm{~T}_{10}\right)$. Similar trend was also observed in case of $\mathrm{N}$ uptake by cotton stalk and it was also found to be on par with the treatment $100 \% \mathrm{NP}+25 \mathrm{~kg} \mathrm{~K} \mathrm{ha}^{-1}+$ biofertilizers $\left(\mathrm{T}_{7}\right)$.

The significantly higher $\mathrm{P}$ uptake (4.02 $\mathrm{kg} \mathrm{ha}^{-1}$ ) by cotton seed was observed with the application of $50 \% \mathrm{~N}$ through gliricidia + $50 \% \mathrm{~N}$ through inorganics + biofertilizers + $100 \% \mathrm{P}+25 \mathrm{~kg} \mathrm{~K} \mathrm{ha}^{-1}\left(\mathrm{~T}_{10}\right)$ and it was on par with the application of $100 \% \mathrm{NP}+25 \mathrm{~kg} \mathrm{~K}$ $\mathrm{ha}^{-1}+$ biofertilizers $\left(\mathrm{T}_{7}\right)$. Similar trend was also observed in case of $\mathrm{P}$ uptake by cotton stalk.

The significantly higher $\mathrm{K}$ uptake $\left(4.27 \mathrm{~kg} \mathrm{ha}^{-1}\right)$ by cotton seed was observed with the application of $50 \% \mathrm{~N}$ through gliricidia + $50 \% \mathrm{~N}$ through inorganics + biofertilizers + $100 \% \mathrm{P}+25 \mathrm{~kg} \mathrm{~K} \mathrm{ha}^{-1}\left(\mathrm{~T}_{10}\right)$ and it was found to be on par with application of $100 \% \mathrm{NP}+$ $25 \mathrm{~kg} \mathrm{~K} \mathrm{ha}{ }^{-1}+$ biofertilizers $\left(T_{7}\right)$. Similar 
trend was also observed in case of $\mathrm{K}$ uptake by cotton stalk.

\section{References}

Badole, S.B. and S.D.More, 2000a. Yield and nutrient uptake as influenced byintegrated nutrient supply system in cotton. J. Indian. Soc. Cotton Improv. 25(3): 161-165.

Udadhe N.N., A. P. Dake, B.M. Lambade and S. B. Jibhkate2013, Effect of different INMs treatments on nutrient uptake of hybrid cotton. Ann. Agric. Res. New series 34 (3): 342-384.

Jackson, M.L., 1973. Soil Chemical Analysis.Prentice Hall Publication Pvt. Ltd., New Delhi, India.
Potkile, S.N., J.T. Kamdi and B. N. Dahatonde, 2004. On farm response of $\mathrm{N}, \mathrm{P}$ and $\mathrm{K}$ on cotton - summer groundnut in Central Vidarbha Region. PKV. Res. J. 28 (1): 40-42.

Raj Dev, A.P.Sharma, PromilaKumari and B.S.Duhan, 2007.Effect of balanced fertilization on seed cotton yield and nutrient uptake by cotton under irrigated condition. J. Cotton Res. Dev.21(1):72-74.

Vyas, M.D., A.K. Jain and R.J. Tiwari, 2003.Long term effect of micronutrients and FYM on yield and nutrient uptake by soybean on TypicChromustert. J. Indian Soc. Soil Sci. 51:45- 47.

\section{How to cite this article:}

Megha S. Khambalkar, V.V. Gabhane and Shilpa V. Khambalkar. 2017. Influence of INM on Nutrient Uptake of Cotton in Dry Land Condition. Int.J.Curr.Microbiol.App.Sci. 6(8): 36423646. doi: https://doi.org/10.20546/ijcmas.2017.608.440 